\section{L'inhibition chimique de NAT10 corrige les défauts des cellules laminopathiques}

Delphine Larrieu ${ }^{1}$, Raphaël Rodriguez ${ }^{2}$, Sébastien Britton ${ }^{3}$
${ }^{1}$ The Wellcome Trust/Cancer Research UK Gurdon Institute and Department of Biochemistry, University of Cambridge, Tennis Court Road, CB2 IQN Cambridge, Royaume-Uni ;

${ }^{2}$ Institut de chimie des substances naturelles, CNRS, Gif-sur-Yvette, France;

${ }^{3}$ Institut de pharmacologie et de biologie structurale, CNRS, Université de Toulouse-Université Paul Sabatier, équipe labellisée Ligue contre le cancer, 31077 Toulouse, France. d.larrieu@gurdon.cam.ac.uk delphine.larrieu@gurdon.cam.ac.uk

\section{La lamina nucléaire maintient} l'architecture du noyau

Chez les eucaryotes, la membrane interne du noyau est bordée d'un maillage de protéines fibrillaires appelé lamina nucléaire, composé des protéines dénommées lamines, incluant les lamines $A$ et $C$ [1]. La lamina nucléaire est un élément crucial du maintien de l'architecture et de la forme du noyau, ainsi que de l'organisation globale de la chromatine, car elle sert de plateforme d'ancrage pour l'hétérochromatine et pour les régions télomériques $[2,3]$. La lamina fait aussi le lien entre le noyau et le cytosquelette car elle interagit avec des protéines transmembranaires telles que SUNI (Sadl and UNC84 domain containing 1) ou les protéines nesprines [4]. Ces propriétés de la lamina expliquent son rôle essentiel dans l'organisation structurelle de la cellule. De ce fait, les mutations des gènes codant pour les lamines sont associées à un large éventail de maladies, regroupées sous le nom de laminopathies [5], dont fait partie le syndrome de vieillissement prématuré Hutchinson-Gilford progeria syndrome (HGPS).

\section{Une mutation sur le gène LMNA cause le syndrome HGPS}

Le syndrome HGPS est très rare et affecte environ un nouveau-né sur quatre millions à travers le monde. Il est causé par une mutation ponctuelle de novo sur un des allèles du gène $\operatorname{LMNA}[6,7]$, responsable de l'expression d'une protéine lamine $A$ tronquée, appelée progérine. À cause de cette mutation, les cellules accumulent une forme toxique de la progérine, modifiée par couplage d'un groupement farnésyl à une cystéine de la lamine (processus de farnésylation). La progérine farnésylée s'incorpore dans la lamina nucléaire où elle a un effet dominant négatif, provoquant la déformation et la lobulation du noyau ainsi qu'une désorganisation globale de la structure chromatinienne, conduisant à la sénescence prématurée [8] et à la mortalité précoce des enfants atteints, à l'âge de 13 ans en moyenne.

\section{Les traitements disponibles}

La forme farnésylée de la progérine étant toxique, des inhibiteurs de farnésylation (FTI: farnesyl transferase inhibitors) ont été testés en clinique chez des enfants atteints de progeria. Ils ont entraîné une modeste amélioration de certains aspects de la pathologie, et une faible augmentation de l'espérance de vie de ces enfants. II n'existe à ce jour aucun autre traitement de cette maladie. C'est pourquoi notre étude s'est focalisée sur l'identification de nouvelles molécules capables d'améliorer les phénotypes cellulaires des fibroblastes issus des patients HGPS. Dans ces cellules, les noyaux sont fortement déformés, et l'organisation de la chromatine affectée. Ainsi, nous avons analysé l'effet de molécules connues pour inhiber des enzymes régulant la structure de la chromatine, les histones désacétylases et les histones acétyltransférases, sur l'amélioration de la forme de ces noyaux et de la compaction de la chromatine $(\rightarrow)$ [12].

$\rightarrow$ Voir la Nouvelle de Catherine Polese et Denis Mottet, page 730 de ce numéro

\section{Identification de la remodelin}

Dans un premier temps, notre approche a été d'évaluer une série de petites molécules organiques naturelles et de synthèse afin d'identifier de manière non biaisée - c'est-à-dire sans préjuger à l'avance d'un mécanisme de correction ou d'une cible prédéfinie - une molécule qui aurait la propriété d'améliorer l'architecture nucléaire des cellules HGPS. Cette approche nous a permis d'identifier une molécule de synthèse dérivée d'un phénylthiazole, le 4-(4-chlorophényl)2-(2-cyclopentylidenehydrazinyl) thiazole (molécule 1) [9]. L'activité modérée et la faible stabilité de cette molécule nous ont incités à étudier les propriétés d'autres analogues. En particulier, nous avons synthétisé un dérivé contenant une fonction « cyano » riche en électrons en remplacement de l'atome de chlore de la molécule 1 initiale (Figure IA). Cette modification a conduit à un dérivé beaucoup plus stable et plus efficace quant à l'amélioration de l'architecture nucléaire et de plusieurs fonctions cellulaires des cellules de patients HPGS (Figure IB-D). Nous avons choisi de nommer cette drogue «remodelin », afin d'illustrer les propriétés spectaculaires qu'elle exerce sur l'architecture nucléaire, la preuve étant faite qu'il est ainsi possible de corriger les laminopathies en mettant à profit des mécanismes de compensations sans lien direct avec les lamines ou leurs mutations. 


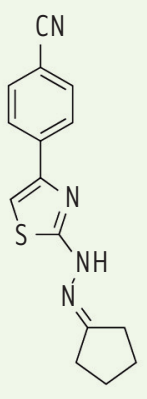

Remodelin
B

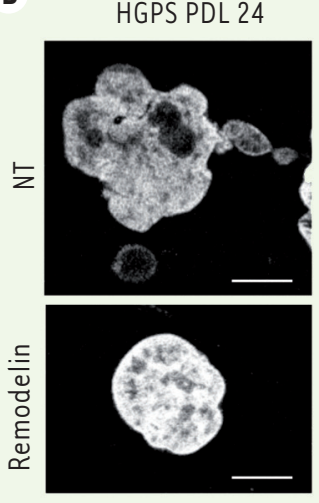

C

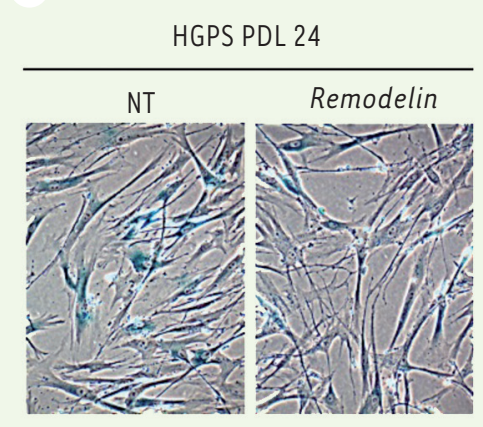

D

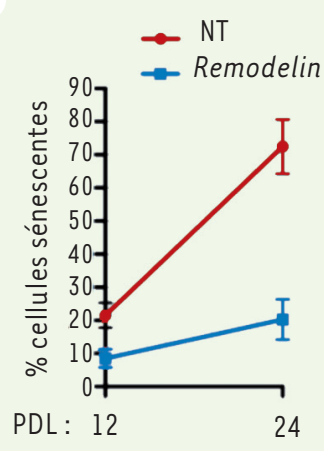

Figure 1. La petite molécule remodelin améliore certains phénotypes des cellules HGPS. A. Structure moléculaire de la remodelin. B. Visualisation de la forme des noyaux des cellules HGPS après 24 passages en culture (PDL 24), par marquage du noyau au DAPI et analyse par immunofluorescence. C. Marquage des cellules sénescentes par essai d'activité $\beta$-galactosidase, et $\boldsymbol{D}$. comptage des cellules positives. NT : non traitées.

Inhiber l'activité acétyl transférase de NAT10 normalise l'organisation nucléaire des cellules laminopathiques

Une forme chimiquement modifiée de la molécule 1 a été synthétisée et utilisée comme «canne à pêche » moléculaire afin d'isoler les protéines associées, ensuite identifiées par spectrométrie de masse [10]. Parmi celles-ci, seule NAT10 (N-acétyltransférase 10) porte un domaine acétyltransférase susceptible d'être inhibé par la molécule 1 et son dérivé, la remodelin. NAT10 comprend un domaine amino-terminal à activité ARN hélicase dépendante de I'ATP, et un domaine carboxy-terminal de la famille de l'histone acétyltransférase GCN5, dont l'activité dépend de l'acétyl coenzyme A (Figure 2A). NAT10 est capable d'acétyler in vitro les histones et la tubuline. Les dimères de tubuline s'assemblent de façon dynamique pour former des filaments, les microtubules, composants du cytosquelette qui déterminent la forme cellulaire et sont nécessaires à la ségrégation des chromosomes durant la mitose. En remplaçant la protéine NAT10 endogène par une forme sauvage ou une forme mutée dans le domaine acétyl-transférase, nous avons pu montrer que bloquer l'activité acétyl-transférase de NAT10 dans les cellules laminopathiques permet de restaurer l'organisation nucléaire dans ces cellules. Ces résultats indiquent que la remodelin améliore le phénotype des cellules laminopathiques en bloquant l'activité acétyltransférase de NATIO [10].

\section{L'activité de NAT10 stimule l'ancrage des microtubules}

II a été montré précédemment que les microtubules exercent une force sur le noyau, déterminant ainsi sa forme [11]. Compte tenu du lien existant entre NAT10 et les microtubules, nous avons analysé l'impact de l'inhibition de NATIO sur l'organisation du cytosquelette de microtubules. Ainsi, nous avons observé qu'inhiber NAT10 en utilisant la remodelin, des siARN ou encore un mutant catalytiquement inactif, perturbe le cytosquelette de microtubules qui apparaissent plus courts et désorganisés (Figure 2B). Afin de tester si l'inhibition de NAT10 normalise l'organisation nucléaire en bloquant la polymérisation des microtubules, nous avons testé l'impact d'inhibiteurs connus de la polymérisation des microtubules tels que le nocodazole. Tout comme la remodelin, le nocodazole permet d'améliorer l'organisation nucléaire des cellules laminopathiques. Toutefois, au contraire du nocodazole, la remodelin ne bloque pas la progression en mitose des cellules, suggérant qu'elle n'est pas un inhibiteur conventionnel de la polymérisation des microtubules. Par ailleurs, en analysant la dynamique de repolymérisation des microtubules, nous avons montré que l'activité acétyl-transférase de NAT10 ne contrôle pas l'initiation de leur polymérisation, mais qu'elle est requise pour leur élongation et leur ramification. Nous proposons que la lamina se comporte comme un rempart isolant le noyau cellulaire et son contenu, en particulier les chromosomes, des tensions générées par les microtubules. Lorsque la lamina est désorganisée, comme chez les patients atteints de progeria, cette force exercée par les microtubules contribue à la déformation du noyau et de son contenu, conduisant à l'accumulation de dommages de l'ADN (Figure 2C). Ainsi, l'inhibition de NAT10 restaure l'organisation nucléaire en limitant les tensions exercées sur le noyau par les microtubules.

Avantages d'une thérapie basée sur la remodelin par rapport à d'autres stratégies

La remodelin offre deux avantages supplémentaires par rapport aux stratégies existantes basées sur l'utilisation des FTI. Le premier est qu'elle est, à notre connaissance, la seule molécule capable à ce jour de réduire de manière 


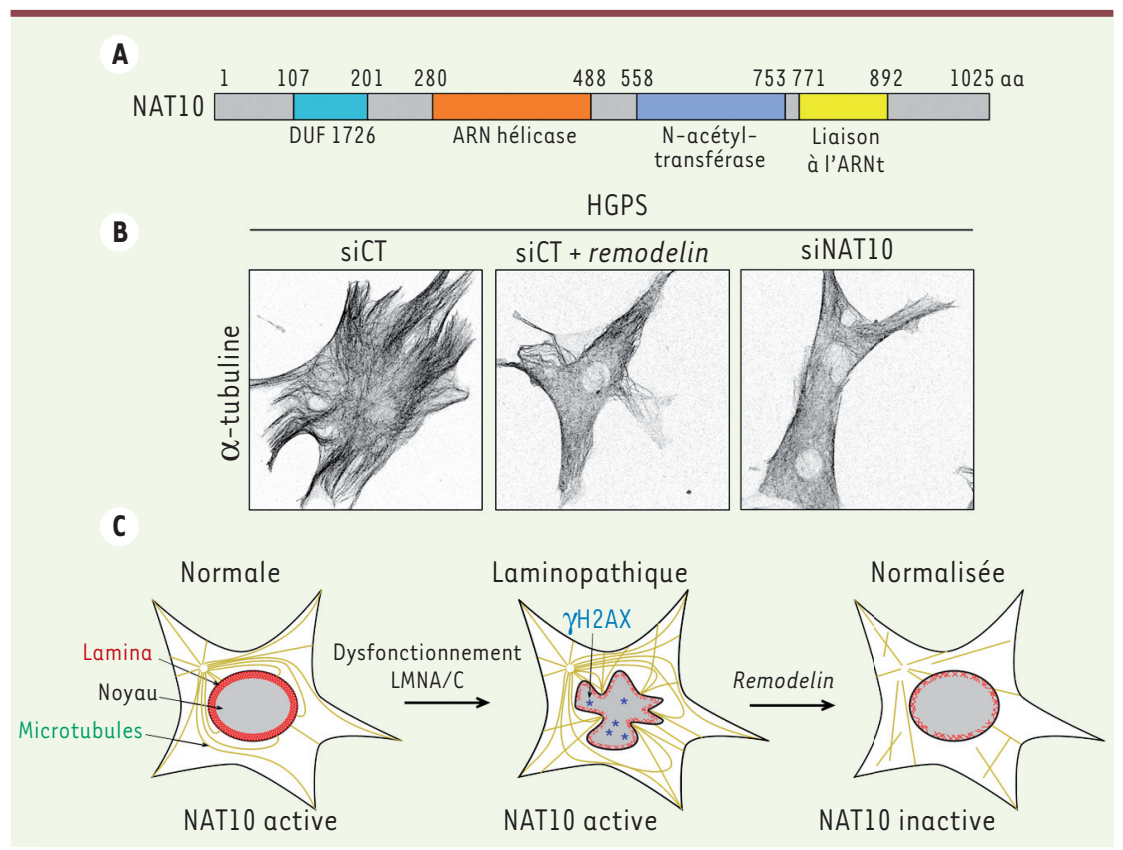

Figure 2. NAT10 est la cible de la remodelin responsable de l'amélioration des phénotypes cellulaires des cellules HGPS. A. Représentation de NAT10 et de ses domaines. B. L'inhibition de NAT10 par la remodelin ou par transfection de siARN contre NAT10 (siNAT10) conduit à la désorganisation du réseau de microtubules. C. Modèle du mode d'action de la remodelin. siCT : siARN contrôle.

significative les dommages endogènes de l'ADN présents dans les cellules issues de patients HGPS. L'accumulation de tels dommages pouvant contribuer au processus de vieillissement prématuré, nous pensons que la remodelin devrait donc permettre d'améliorer certains phénotypes des HGPS. D'autre part, si les FTI ont un effet bénéfique sur les cellules accumulant la progérine, ils ont l'effet inverse sur les cellules normales, conduisant à l'apparition de déformations du noyau et donc à de potentiels effets indésirables à long terme. Au contraire, non seulement nous n'avons pas observé de toxicité liée au traitement de cellules normales avec la remodelin, mais, de plus, les défauts de l'architecture nucléaire causés par les FTI sur ces cellules normales sont bloqués par la remodelin. II serait donc envisageable d'utiliser chez les patients une combinaison de plusieurs inhibiteurs, pouvant permettre d'attaquer les défauts des cellules HGPS sur plusieurs fronts, et ainsi d'espérer un bénéfice plus important.

\section{Perspectives}

Étant donné les résultats très encourageants obtenus en culture sur les cellules issues de patients HGPS, l'étape suivante sera d'évaluer les effets de la remodelin dans des modèles murins. Il existe en effet plusieurs modèles de souris reproduisant les caractéristiques du syndrome HGPS humain, dont un modèle développé par le groupe de Carlos Lopez-0tin [12] dans lequel le gène sauvage LMNA a été remplacé par un allèle mutant portant la mutation c.1827C>T;p.Gly609Gly, qui est l'équivalent de la mutation c.1824C>T;p. Gly608Gly responsable du syndrome HGPS chez l'homme. Ces souris présentent des signes de vieillissement prématuré seulement trois semaines après leur naissance, développent des problèmes cardiaques et ne vivent en moyenne que 120 jours contre deux ans en moyenne pour les souris sauvages. Notre but est d'évaluer la capacité de la remodelin à améliorer les symptômes de vieillissement chez ces souris, mais aussi de déterminer si un tel traitement permettrait d'augmenter leur espérance de vie. $\diamond$
Chemical inhibition of NAT10 corrects defects of laminopathic cells

\section{LIENS D'INTÉRÊT}

Les auteurs déclarent n'avoir aucun lien d'intérêt concernant les données publiées dans cet article.

\section{RÉFÉRENCES}

1. Bridger JM, Foeger N, Kill IR, Herrmann H. The nuclear lamina. Both a structural framework and a platform for genome organization. FEBS J $2007 ; 274: 1354$

2. Dechat T, Pfleghaar $K$, Sengupta $K$, et al. Nuclear lamins: major factors in the structural organization and function of the nucleus and chromatin. Genes Dev 2008; 22 : 832-53.

3. Gonzalez-Suarez I, Redwood AB, Perkins SM, et al. Novel roles for A-type lamins in telomere biology and the DNA damage response pathway. Embo J 2009 ; 28 2414-27

4. Crisp M, Liu Q, Roux K, et al. Coupling of the nucleus and cytoplasm: role of the LINC complex.J Cell Biol $2006 ; 172: 41-53$

5. Worman HJ, Bonne G. Laminopathies: a wide spectrum of human diseases. Exp Cell Res 2007 ; 313 : 2121-33.

6. De Sandre-Giovannoli A, Bernard R, Cau P, et al. Lamin a truncation in Hutchinson-Gilford progeria. Science $2003 ; 300: 2055$

7. Eriksson M, Brown WT, Gordon LB, et al. Recurrent de novo point mutations in lamin $A$ cause Hutchinson-Gilford progeria syndrome. Nature 2003; 423: 293-8.

8. Cohen TV, Hernandez L, Stewart CL. Functions of the nuclear envelope and lamina in development and disease. Biochem Soc Trans 2008 ; 36 : 1329-34.

9. Chimenti F, Bizzarri B, Maccioni $\varepsilon$, et al. A nove histone acetyltransferase inhibitor modulating Gcn5 network: cyclopentylidene- [4-(4'-chlorophenyl) thiazol-2-yl] hydrazone.J Med Chem 2009; 52 : 530-6

10. Larrieu D, Britton S, Demir M, Rodriguez R, Jackson SP. Chemical inhibition of NAT10 corrects defects of laminopathic cells. Science 2014 ; 344 : 527-32.

10. Tamiello C, Kamps MA, van den Wijngaard A, et al. Soft substrates normalize nuclear morphology and prevent nuclear rupture in fibroblasts from a laminopathy patient with compound heterozygous LMNA mutations. Nucleus 2013; 4:61-73.

11. Osorio FG, Navarro CL, Cadiñanos J, et al. Splicingdirected therapy in a new mouse model of human accelerated aging. Sci Transl Med 2011 ; 3 : 106ral07.

12. Polese C, Mottet D. Inhiber HDAC5 : un outil pour couper court à l'immortalité des cellules cancéreuses. Med Sci (Paris) 2014 ; $30: 730-2$.
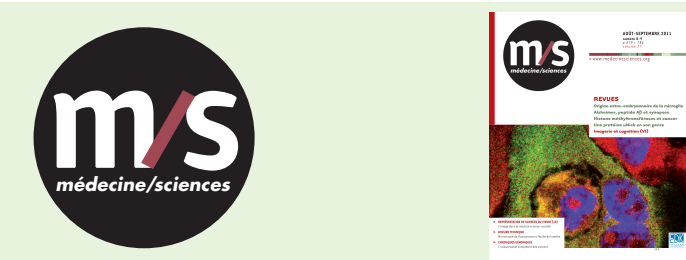

Abonnez-vous à médecine/sciences

Bulletin d'abonnement page 813 dans ce numéro de $\mathrm{m} / \mathrm{s}$ 